\title{
Computation of Microdosimetric Distributions for Small Sites*
}

\author{
D. Chmelevsky and A. M. Kellerer
}

Institut für Medizinische Strahlenkunde der Universität Würzburg, Versbacher Landstraße 5, D-8700 Würzburg, Federal Republic of Germany

\begin{abstract}
Summary. Object of this study is the computation of microdosimetric functions for sites which are too small to permit experimental determination of the distributions by Rossi-counters. The calculations are performed on simulated tracks generated by Monte-Carlo techniques.

The first part of the article deals with the computational procedure. The second part presents numerical results for protons of energies $0.5,5,20 \mathrm{MeV}$ and for site diameters of $5,10,100 \mathrm{~nm}$.
\end{abstract}

\section{Introduction}

Topic of this article is the calculation of microdosimetric distributions. The calculations are performed for heavy charged particles and for spherical regions whose diameter is between 1 and $100 \mathrm{~nm}$. The input data on which the calculations are based are simulated tracks of heavy charged particles. The simulated tracks have been generated by Paretzke $[12,13]$ on the basis of known and interpolated crosssections of charged particles in low atomic weight media ${ }^{1}$.

The general properties of microdosimetric quantities and their distributions have been considered earlier $[7,8]$. The theoretical background need therefore not be repeated. The microdosimetric variable which will be used in the following is the lineal energy, $y$. This facilitates comparison with the familiar concept of LET. The connection to the other microdosimetric variables energy imparted, $\varepsilon$, and specific energy, $z$, is straightforward. Numerical results will only be given for the single event distributions. This is sufficient since the dose dependent distributions can be derived from the single event distributions.

* This investigation was supported by Public Service Research Grants No. CA 12536 and CA 15307 from the National Cancer Institute and by Research Contract 208-76-7 BIO D of Euratom

1 A recent survey of the subject of collision cross-sections is found in the Proceedings of the Symposium on the Distributions of Secondary Electrons from Ionizing Collisions [Radiat. Res. 63 (1975)]. Valuable information is given in earlier work $[2,3,6,10]$ 
The first part of this article deals with the computational procedures. The second part contains numerical results. These are mainly restricted to single event distributions for protons of energy between $500 \mathrm{keV}$ and $20 \mathrm{MeV}$.

\section{Computational Procedure}

\section{Principle of the Approach}

The computations are based on simulated particle tracks. Each simulated particle track is represented by the so-called inchoate distribution. As pointed out earlier [7] the inchoate distribution is given by the coordinates of the transfer points, i.e. of those points where the primary or secondary ionizing particles transfer energy to the medium. The simulated charged particle tracks are those obtained by Monte Carlo simulation $[12,13]$. In principle such particle tracks might also be derived from experimental observations for example in cloud chambers [4]. The computational procedures are applicable regardless of the origin of the input data.

In the experiment one exposes one spherical cavity, the Rossi counter, to a sufficiently large fluence of charged particles. This is natural, since the instrumentation is costly while tracks can be generated abundantly. In the computations the situation is reversed, since it is wasteful to generate a great number of tracks. It is therefore important that the information in each simulated track be fully exhausted. An essential point is accordingly the development of efficient sampling routines. The topic will be treated in some detail, since it is of interest even beyond the particular application presented in this study.

It will be found sufficient to use short segments of the tracks of heavy charged particles which contain one or a few thousand ionizations. As in the earlier discussion [9] the term short segment implies that the LET of the particle does not change significantly along this segment and that the segment is straight. The numerical procedure will, however, be applicable regardless of these conditions.

\section{Method of Sampling}

The following discussion will make use of an important simplification. Only the ionizations in the simulated particle tracks are accounted for, while the excitations are disregarded. This means that the particle track is reduced to those transfer points which stand for ionizations. Furthermore the energy transfer belonging to each of the remaining transfer points is set equal to $W$, the mean energy expended per ionization. This is a drastic simplification which may not always be acceptable. However it is advantageous insofar as numerical results obtained in this way are comparable to experimental data based on ion collection in gas. A second advantage is a substantial reduction of the input data and a simplification of the numerical calculations. The discussion of the sampling procedures will make use of the same simplification. The formalism can however be readily adapted to the more rigorous analysis where charged particle tracks are evaluated in their precise form with all 
transfer points and with all the proper energy transfers assigned to these transfer points.

If one considers a spherical site of radius $r$, the associated volume of the charged particle track is that volume which results if each transfer point, i.e. each ionization, is made the center of a sphere of radius $r$. The individual spheres will, for the purpose of the present discussion, be called associated spheres; this will help to avoid confusion of these spheres with the sphere which is the reference site for the determination of the microdosimetric variable $y$. If one selects a point inside the associated volume and considers a sphere of radius $r$ centered at this point, then this sphere will always contain at least one transfer point. If one considers a point outside the associated volume and a sphere of radius $r$ centered at this point, then this sphere will never contain a transfer point. It is therefore sufficient to consider only those spheres whose centers are inside the associated volume, i.e. it is sufficient to sample points inside the associated volume.

The associated volume is made up of the associated spheres, and these may partially overlap. The microdosimetric variables $y, \varepsilon$, or $z$ are largest at those points where the degree of overlap is largest. If, at a certain sampling point, $n$ associated spheres overlap then the value of $y$ is:

$$
y=\frac{3 m W}{4 r} .
$$

The equation is based on the simplification which has been mentioned above, i.e. it results if each transfer point is assumed to be an ionization which corresponds to an energy transfer $W$.

According to these considerations it is sufficient to calculate the fractions of the associated volume which correspond to different degrees of overlap of the associated spheres. This would seem to be an elementary geometrical problem once the coordinates of the transfer points are known. Upon further reflection one finds however that a rigorous solution of the problem is far too complicated to be feasible.

One is therefore forced to use sampling procedures. The straightforward method is the random choice of a sufficient number of sampling points inside the associated volume. This method will not actually be used since a procedure exists which is far more efficient for straight particle tracks. It is nevertheless of interest to note that the selection of points in the associated volume need not be uniform, and that, in fact, a uniform selection would be difficult due to the generally complicated shape of the associated volume. However one can use the method that has been designated as sampling over individual tranfers [8]. This is a two-step method. First one selects randomly a transfer point, then one selects randomly a point within the associated sphere, i.e. within the sphere centered at this transfer point. The resulting point is used as sampling point at which the microdosimetric variable $y$ is determined, and the procedure is repeated until a sufficient number of points has been sampled. The method is practicable; it may indeed be the method of choice in the case of electron tracks which, due to their curvature, are more complicated than the tracks of heavy charged particles. For heavy charged particles however one reduces the computational work greatly if one samples the microdosimetric variables not on individual points but on straight lines which are parallel to the particle track. The procedure will be described in the following. 


\section{Sampling Procedure Applicable to Straight Particle Tracks}

As pointed out in the preceding section it would be difficult to calculate analytically the fractions of the associated volume which correspond to different multiplicities of overlap, i.e. to the different values of the microdosimetric variable $y$. On the other hand, it is rather inefficient to use the simple alternative of determining the multiplicity of overlap, i.e. the value of $y$, at individual sampling points. There is however the possibility of an intermediate approach. Such an intermediate approach is to calculate the distribution of the microdosimetric variable in randomly selected linear subspaces, such as planes or straight lines. If planes were to be used, one would have to calculate the areas of overlap in a cluster of circles which result when the associated spheres are intersected by the plane. The problem is still too complicated for numerical evaluation. For straight lines, on the other hand, the situation is acceptable; here one deals with the problem of determining distances of overlap of a cluster of intervals which result from the intersection of the straight line with the associated spheres. Accordingly the calculations are done by selecting randomly straight lines and by calculating the distribution of $y$ on these sampling lines.

The sampling of the microdosimetric variable $y$ on straight lines instead of individual points has the advantage of generating considerably more information per sampling operation. The method represents an efficient combination of the analytical and the Monte Carlo approach; it is particularly useful if one deals with simulated segments of heavy charged particle tracks. The reason is that these track segments are straight or approximately straight, and that one can therefore use sampling lines parallel to the track core; in this way one obtains a considerable intersection of each such line with the associated volume.

In each calculation one chooses successively a large number, $N$, of sampling lines. Typically the number will be 1000 or 2000 . For each line one obtains a distribution, $f_{v}(y),(v=1,2 \ldots N)$, of the values of lineal energy $y$. This is the distribution of values $y$ for all the spheres of radius $r$ which have their center on the $v$-th sampling line. The overall distribution of $y$ for the track segment is then obtained by superposition of the $N$ distributions $f_{\nu}(y)$. The following sections will deal with the selection of sampling lines, with the proper weight factors in the superposition of the individual distributions, and with the calculation of these individual distributions $f_{v}(y)$.

\section{Selection of Sampling Lines}

Each sampling line has a certain length of intersection with the associated volume. Let $l_{v}$ be this length of intersection for the $v$-th sampling line. This is the proper statistical weight of the function $f_{v}(y)$ in its contribution to the overall distribution $f(y)$.

If the selection probability of the $v$-th sampling line is not proportional to its statistical weight, $l_{v}$, then appropriate compensation factors, $c_{v}$, must be used in the superposition which leads to $f(y)$ :

$$
f(y)=\sum_{\nu=1}^{N} c_{\nu} f_{v}(y) .
$$


It has been pointed out earlier (see p. 209 of Ref. 8) that the compensation factor, $c_{v}$, must be equal to the ratio of the statistical weight, i.e. $l_{v}$, and the selection probability $p_{v}$. In order to derive the proper factors $c_{v}$ one must therefore determine the probabilities, $p_{v}$, which belong to the selection procedure which is used.

An efficient method to select sampling lines is closely connected to the sampling over individual transfers. In the procedure one first selects randomly a transfer point. Then one selects randomly a point in the associated sphere. A line through this point which is parallel to the track segment is then taken as the sampling line.

One can readily see that the selection probability, $p_{v}$, of a sampling line is, in this case, proportional not to $l_{v}$ but to the total length, $L_{v}$, which is obtained if one sums the intersections of the line with the individual associated spheres separately. The length $L_{v}$ is always equal or larger than $l_{v}$; it is in fact equal to $l_{v}$ multiplied by the mean multiplicity of overlap on the intersection.

In order to obtain the proper statistical weights one must therefore average the functions $f_{v}(y)$ for the individual sampling lines according to the following formula:

$$
f(y)=\sum_{\nu=1}^{N} \frac{l_{v}}{L_{v}} f_{v}(y) / \sum_{\nu=1}^{N} \frac{l_{v}}{L_{v}} .
$$

It will be seen that this expression reduces to a simple form in the actual numerical evaluation.

In practice the selection procedure for the sampling lines is slightly modified in order to reduce the Monte Carlo component of the approach. Typical track segments which are used for the calculations consist of 1000 or 2000 transfer points which represent ionizations. In the sampling procedure these transfer points are not randomly selected but are selected consecutively, and one sampling line is established for each of the transfer points by choosing a point in the associated sphere belonging to the transfer point. This leads usually to sufficient accuracy. The formula for the selection of a sampling line which traverses the associated sphere of a transfer point is given in the appendix.

The next section deals with the calculation of the distributions $f_{v}(y)$ on the individual sampling lines.

\section{Computation of the Distributions}

Consider a Cartesian coordinate system with the $z$-axis on the core of the track segment. The sampling line is parallel to the $z$-axis and can therefore be characterized by its coordinates $x_{0}$ and $y_{0}$. The coordinates of the transfer point $T_{i}$ ( $i=$ $1, \ldots K)$ are designated by $x_{i}, y_{i}$, and $z_{i}$. For each transfer point which is a distance less than $r$ from the sampling line one can compute the interval where the sampling line intersects the associated sphere of that transfer point. The $z$-coordinates, $z_{s}$ and $z_{t}$, of the starting point and the terminating point of the interval are obtained by the formula:

$$
z_{s}, z_{t}=z_{i} \pm \sqrt{r^{2}-\left(x_{i}-x_{0}\right)^{2}-\left(y_{i}-y_{0}\right)^{2}} .
$$



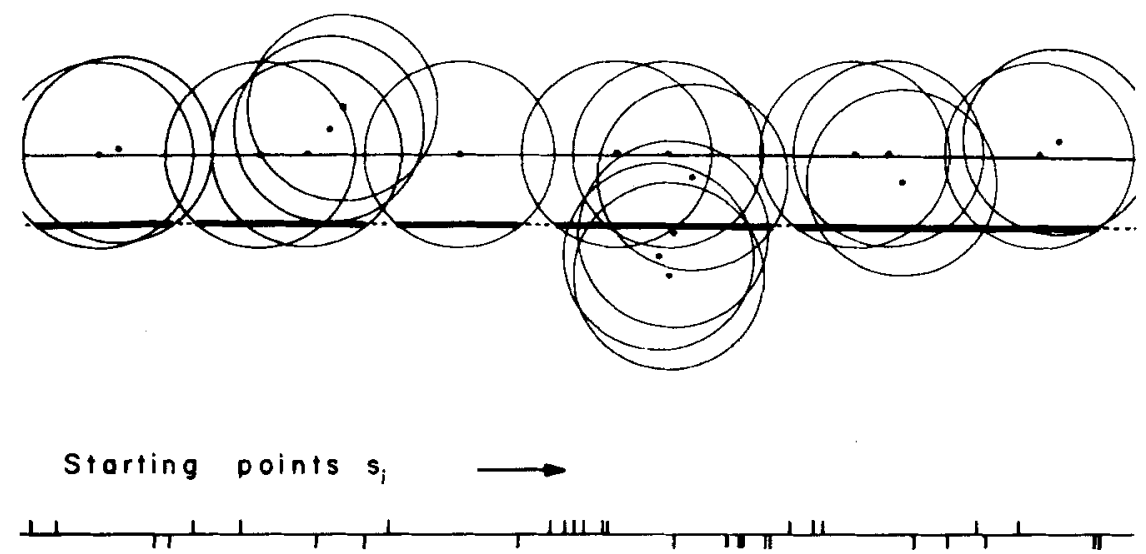

Terminating points

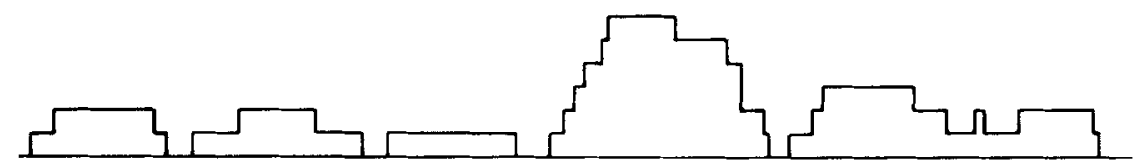

Profile of lineal energy on line segment

Fig. 1. Two-dimensional diagram to illustrate the computation of the functions $f_{v}(y)$ from track segments of heavy particles. The upper part is a schematic diagram of a track segment with its associated volume. The heavy line segments are those parts of the sampling line where $y$ is not zero. The two lower parts of the figure illustrate the two steps in the computation as they are discussed in the text

For a given sampling line one calculates all the coordinates $z_{s}$ and $z_{t}$ of the starting and the terminating points. The situation is represented schematically in Figure 1. If $n$ is the number of intervals of intersection one obtains $2 n$ points. After these points are computed they are ordered into one common array so that their $z$-coordinates are in increasing order. One thus obtains a new array $z_{i}^{\prime},(i=1,2, \ldots 2 n)$. Its components $z_{i}^{\prime}$ are the $z$-coordinates of the $2 n$ points. A second array, $m_{i},(i=1$, $2, \ldots 2 n)$, has the values $m_{i}=1$ or $m_{i}=-1$ depending on whether the $i$-th point is the starting point or the terminating point of an interval.

The multiplicity of overlap in the interval from $z_{i}^{\prime}$ to $z_{i+1}^{\prime}$ is equal to

$$
M_{i}=\sum_{j=1}^{i} m_{j} .
$$

This quantity corresponds to the function represented on the bottom of Figure 1.

It is now easy to obtain that length, $s_{v}(M)$, on the sampling line which corresponds to the multiplicity $M$ of overlap. One merely has to sum the lengths of those segments for which $M_{i}=M$. The normalized distribution of multiplicities on the line is obtained as

$$
f_{v}(M)=s_{v}(M) / \sum_{M^{\prime}=1}^{M_{\max }} s_{\nu}\left(M^{\prime}\right)=s_{v}(M) / l_{v} .
$$


The multiplicity $M$ is proportional to the lineal energy $y$, and is used here since it is the quantity actually appearing in the computation. However the change to $y$ [see Eq. (1)] is trivial, and one can therefore write the corresponding equation in terms of $y$ :

$$
f_{v}(y)=s_{v}(y) / l_{v} .
$$

Inserting this into Equation (3) one obtains a simple relation which is the basis of the numerical computations:

$$
f(y) \sim \sum_{v=1}^{N} s_{v}(y) / L_{v} .
$$

The term $s_{v}(y)$ is that length on the $v$-th sampling line which belongs to the value $y$ of the lineal energy.

In order to avoid errors due to wrong values of $y$ near the end of the track segments one disregards in the analysis those parts of the sampling lines which are within the length $r$ of the endpoints of the segment. The resulting change in some of the formulae is elementary.

\section{Numerical Results}

As stated earlier, the computations use as input data track segments which are the result of Monte-Carlo simulation ${ }^{2}[12,13]$. The present status of the method of generating simulated charged particle tracks is, at least partly, documented in the literature $[12-14,20]$. The validity of the data and the remaining uncertainty due to incomplete knowledge of the differential cross sections for electronic collisions are not the topic of this article; the procedures described in the preceding sections will remain valid regardless of future changes in the input data.

Even in the presence of systematic uncertainties a survey of numerical data will be useful to the radiobiologist. The results will illustrate correctly the characteristic dependence of the microdosimetric distributions on site diameters and stopping powers.

The simulated tracks which have been utilized are short segments containing a minimum of 1000 ionizations. This corresponds to a length of about $0.7 \mu \mathrm{m}$ for $500 \mathrm{keV}$ protons and about $12 \mu \mathrm{m}$ for $20 \mathrm{MeV}$ protons. Only sites with radius up to $100 \mathrm{~nm}$ are considered and the change of LET of a particle traversing such sites is insignificant. The LET is therefore taken to be constant on all track segments. Due to statistical fluctuations the track segments do not correspond exactly to the underlying LET values. All track segments have, however, been adjusted to the LET values for water [5].

Adjustment of the LET for a given track segment is a relatively simple operation. One merely rearranges the individual deltas along the $z$-axis; the term delta is used to designate, in the present context, individual ionizations, groups of ioniza-

2 A more complete compilation of numerical results is to be found in [1]. Some results for considerably larger sites have been obtained in an earlier study of Wilson [21] which is also based on simulated particle tracks 


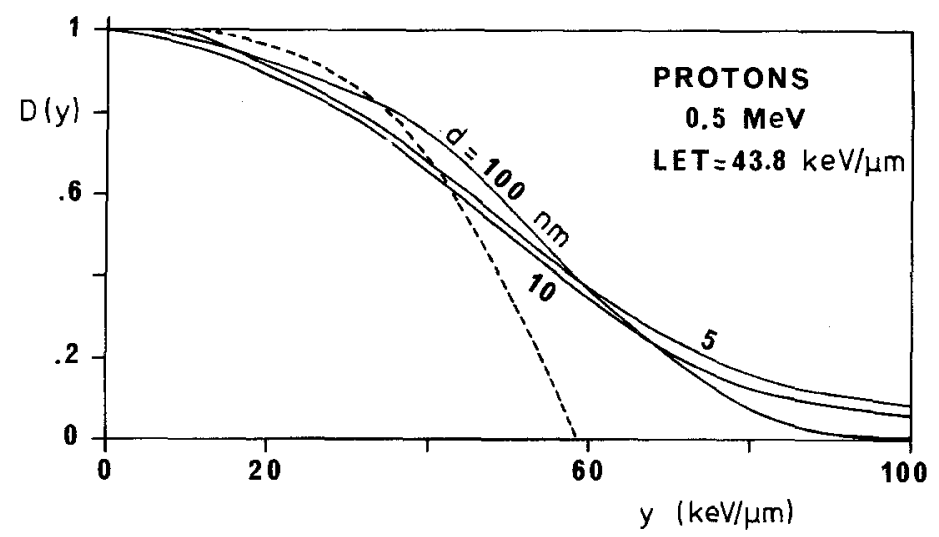

Fig. 2. Sum distribution, $D(y)$, of absorbed dose in lineal energy for protons of $0.5 \mathrm{MeV}$ in spheres of diameter 5,10 and $100 \mathrm{~nm}$. The broken line represents the function which corresponds to the approximation in terms of the LET-concept

tions, or delta rays resulting from one electronic collision of the primary particle. The procedure is of practical importance not only because it permits minor adjustments of LET. It also makes it possible to transform a simulated proton track into the track of a heavier ion of the same velocity. Results for heavier ions are however not the topic of the present article.

The results are subject to two types of statistical uncertainty. The first is due to the fact that each track contains only a limited number of deltas. This error is most significant for the $20 \mathrm{MeV}$ protons where delta rays up to about $40 \mathrm{keV}$ can be generated, but occur with only small frequency. It has however been found that occurrence or non-occurrence of such high energy delta rays is of only moderate influence on the results. The reason is that the distributions of $y$ produced by the fast protons and by relatively fast electrons are similar for the small regions which are here considered.

The second error is due to the use of only a limited number of sampling lines. This error is small and can be quantitatively assessed by comparing the mean value $\bar{y}_{D}$ of the explicit distribution $f(y)$ with the value $\bar{y}_{D}$ which is calculated directly from the simulated track according to a formula derived in an earlier article [9]. The difference was in all cases less than $1 \%$. This error is much smaller than the systematic uncertainty in the simulated tracks. A high precision is nevertheless desirable in order to make results for different site diameters comparable.

Figures 2-4 represent the dose distribution, $D(y)$, of the lineal energy for proton energies of $0.5,5$, and $20 \mathrm{MeV}$. In each figure the distributions are given for sites of diameter 5, 10 and $100 \mathrm{~nm}$. The dose distribution $D(y)$ is here defined as (see also [8]).

$$
D(y)=\int_{y}^{\infty} d\left(y^{\prime}\right) d y^{\prime}=\frac{1}{\bar{y}_{F}} \int_{y}^{\infty} y^{\prime} f\left(y^{\prime}\right) d y^{\prime} .
$$

$D(y)$ is equal to the fraction of energy which is delivered in events of lineal energy exceeding $y$. One track segment for each of the proton energies is used in the computations. 


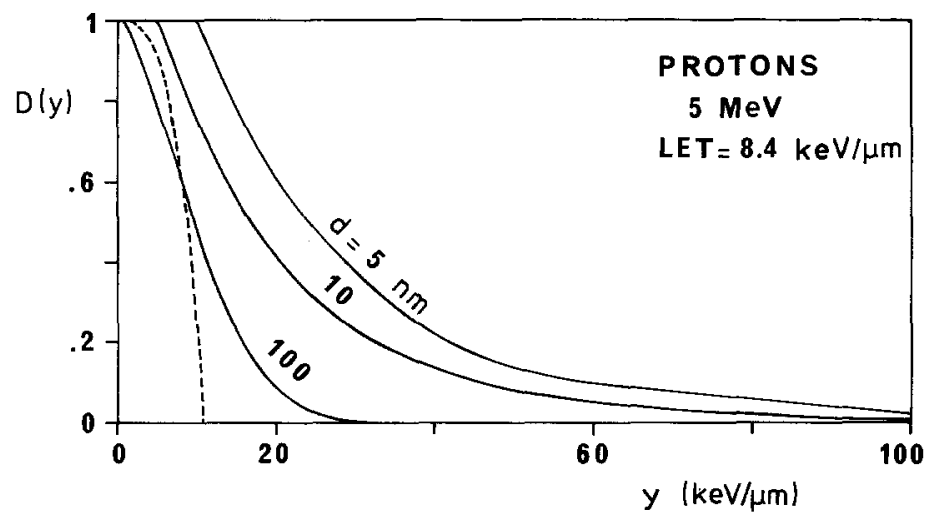

Fig. 3. Sum distribution, $D(y)$, of absorbed dose in lineal energy for protons of $5 \mathrm{MeV}$ in spheres of diameter 5, 10 and $100 \mathrm{~nm}$. The broken line represents the function which corresponds to the approximation in terms of the LET-concept

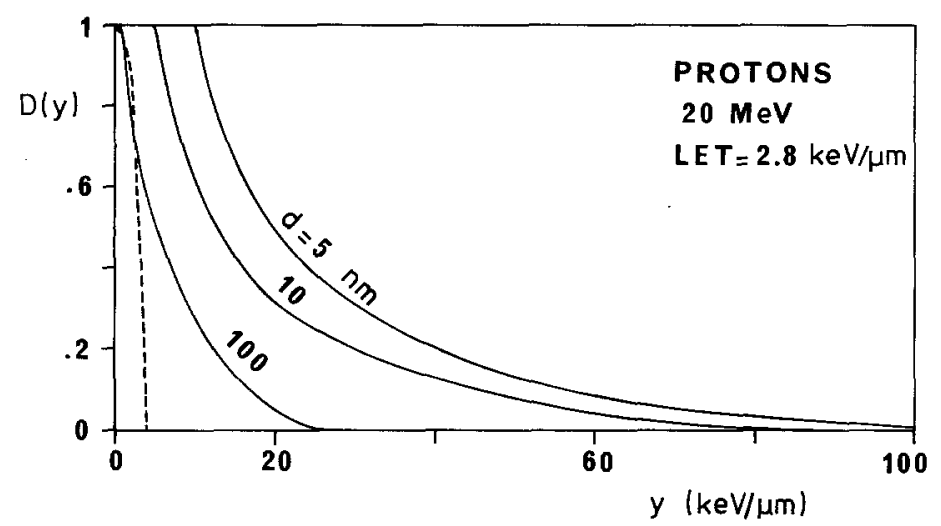

Fig. 4. Sum distribution, $D(y)$, of absorbed dose in lineal energy for protons of $20 \mathrm{MeV}$ in spheres of diameter 5,10 , and $100 \mathrm{~nm}$. The broken line represents the function which corresponds to the approximation in terms of the LET-concept

The distributions should be drawn as step functions, since according to Equation (1) the quantity linear energy is linked to the number, $n$, of ionizations and is therefore a discrete variable. In the interest of clearer representation the functions have however been approximated by continuous lines. Similar functions would result if, in the computations, actual energy transfers and not the average value $W$ were assigned to the transfer points.

The broken lines in Figures 2-4 indicate the distributions which would result from the simple LET concept, i.e. from the assumption, that the particle tracks are straight lines with continuous energy transfer and with no radial extension. One can readily see that the LET concept is only a crude approximation even for the protons of lowest energy and for the largest sites which are here considered. For the protons 


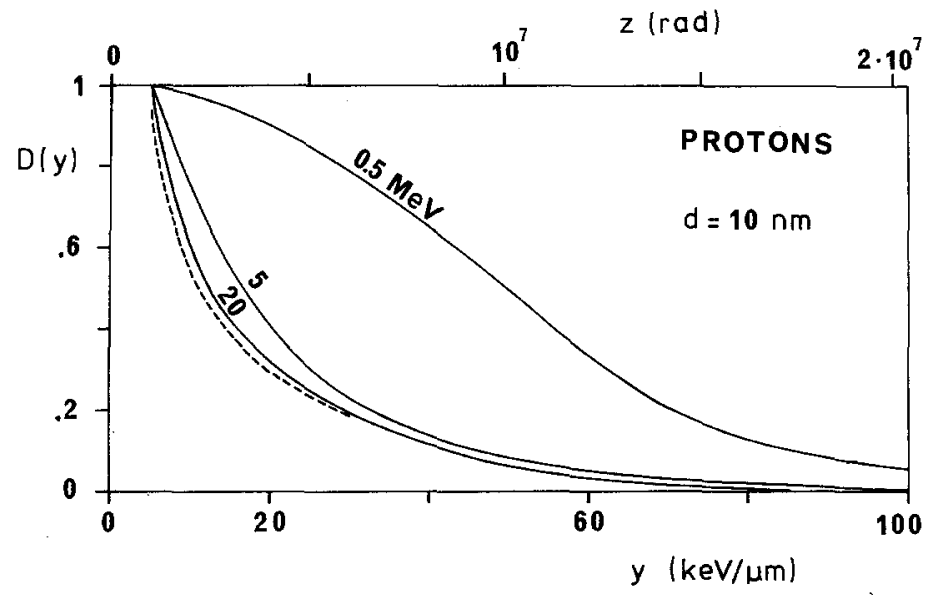

Fig. 5. Sum distribution, $D(y)$, of absorbed dose in lineal energy in a sphere of $10 \mathrm{~nm}$ diameter for protons of $0.5,5$, and $20 \mathrm{MeV}$. The broken line corresponds to electrons of minimum stopping power

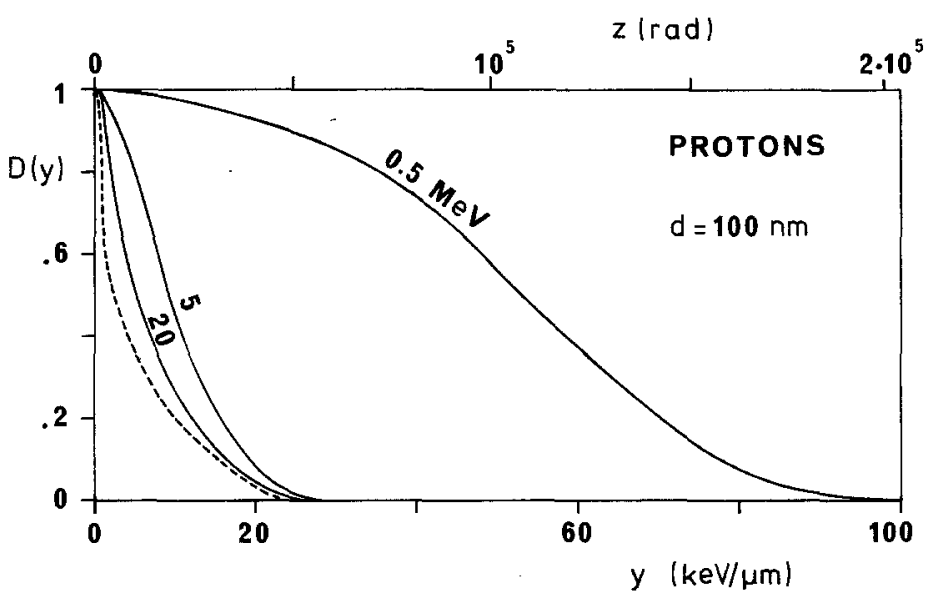

Fig. 6. Sum distribution, $D(y)$, of absorbed dose in lineal energy in a sphere of $100 \mathrm{~nm}$ diameter for protons of $0.5,5$, and $20 \mathrm{MeV}$. The broken line corresponds to electrons of minimum stopping power

of $20 \mathrm{MeV}$, and $5 \mathrm{MeV}$ one realizes that energy concentrations in small sites are much larger than expected on the basis even of the unrestricted stopping power. These energy concentrations are mainly due to the occurrence of individual delta rays in the site.

The dependence of the distributions on the stopping power of the proton is brought out more clearly in Figures 5 and 6. The distributions shown in Figures 2-4 have here been rearranged into two groups belonging to the site diameters $10 \mathrm{~nm}$ and $100 \mathrm{~nm}$. It is of particular interest to observe that a threefold change of LET 


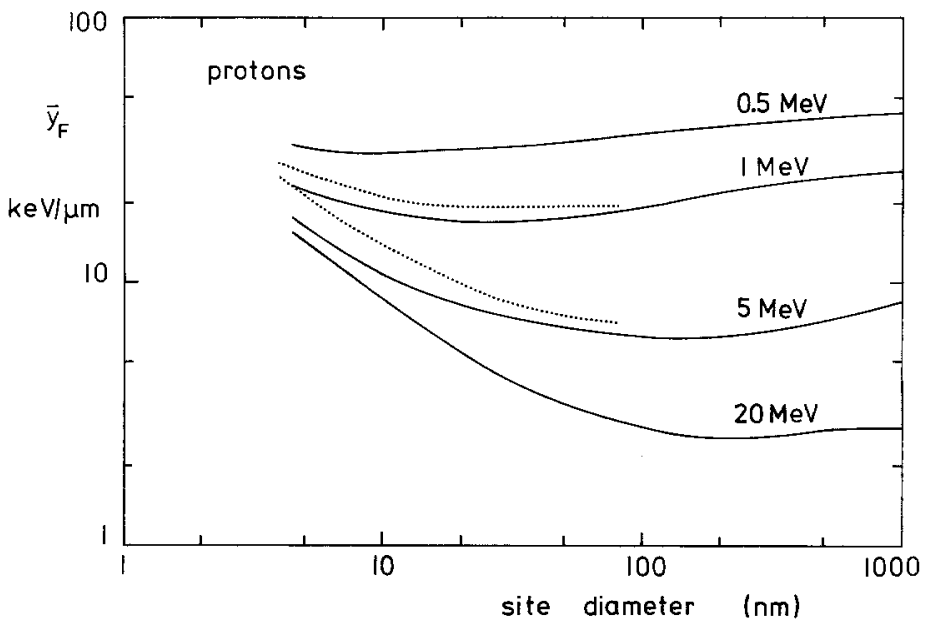

Fig. 7. Frequency mean, $\bar{y}_{F}$, as a function of site diameters for protons of energy $0.5,1,5$, and $20 \mathrm{MeV}$. The values for site diameters exceeding $100 \mathrm{~nm}$ are extrapolated

Table 1. Values of $\bar{y}_{D}$ in $\mathrm{keV} / \mu \mathrm{m}$ and of $\zeta$ in rad (lower numbers, in brackets) for protons of different energies and for spherical sites of different diameters. The stopping power is for water [5]; its values are indicated in Figures 2-4

\begin{tabular}{clll}
\hline $\begin{array}{l}\text { Site diameter } \\
(\mathrm{nm})\end{array}$ & \multicolumn{2}{l}{ Proton energy $(\mathrm{MeV})$} \\
\cline { 2 - 4 } & 0.5 & 5 & 20 \\
\hline 5 & 50 & 26.7 & 23.8 \\
& $\left(40.7 \times 10^{7}\right)$ & $\left(2.17 \times 10^{7}\right)$ & $\left(1.94 \times 10^{7}\right)$ \\
10 & 50 & 20.3 & 17.4 \\
& $\left(1.02 \times 10^{7}\right)$ & $\left(4.13 \times 10^{6}\right)$ & $\left(3.54 \times 10^{5}\right)$ \\
100 & 52 & 10.3 & 7 \\
& $\left(1.06 \times 10^{5}\right)$ & $\left(2.10 \times 10^{4}\right)$ & $\left(1.43 \times 10^{4}\right)$ \\
\hline
\end{tabular}

from the $5 \mathrm{MeV}$ proton to the $20 \mathrm{MeV}$ proton leads to only a moderate shift in the microdosimetric distributions.

The broken lines in Figures 5 and 6 are approximations of the distributions for fast electrons. Since no tracks of fast electrons were available, these have been simulated by transforming the track of the $20 \mathrm{MeV}$ proton to a stopping power of $0.2 \mathrm{keV} / \mu \mathrm{m}$. Inspite of the change of stopping power by a factor of 14 the resulting distribution is, in the case of the smaller site, almost identical to that for the fast protons.

In Figures 5 and 6 the quantity specific energy, $z$, is plotted on the upper ordinate. This is useful as it illustrates the extremely large values of the specific energy produced in small sites by particles even of low stopping power. 
Finally the mean values, $\bar{y}_{F}$, corresponding to the distributions are given in Figure 7.

On the basis of the ideas of Rossi and coworkers [15-17, 19] microdosimetry has in recent years developed into a separate field of growing importance. Now, as the study of small sites finds increasing attention [18], the connections to earlier work by Lea [11] become more apparent. A certain comparison is given in Figure 7. The dotted lines in this figure are obtained from values of the associated volumes given by Lea. These data are close to the present results.

The important mean values $\bar{y}_{D}$ are given only in tabular form for the distributions $D(y)$ in Figures $2-6$. A detailed discussion of the quantity $\bar{y}_{D}$ and the socalled distance distribution for protons and heavier ions will be found in a subsequent article.

Acknowledgements. Part of this study has been performed at the Radiological Research Laboratory of Columbia University, New York. We are grateful to Prof. H. H. Rossi whose contribution to the work has been essential. We are also indebted to Dr. N. Parmentier and M. M. Dousset for their active interest, and to Dr. H. Paretzke for providing the simulated particle tracks.

\section{Appendix}

\section{Random Selection of Coordinates}

\section{Selection of a Variable Which Follows a Specified Distribution}

Algorithms for the generation of pseudo-random numbers generally produce a random variable, $u$, which is uniformly distributed in the interval $[0,1]$. The problem then arises how to obtain a suitable function $x(u)$ which follows a specified nonuniform distribution. An answer can best be found if one considers the general case.

If $G(u)$ is the sum distribution of $u$ and $F(x)$ is the sum distribution of $x$, and if $x$ is a monotoneously increasing function of $u$, the following relation holds:

$$
G(u)=P(\underline{u} \leqslant u)=P(x(\underline{u}) \leqslant x(u))=P(\underline{x} \leqslant x)=F(x) .
$$

Therefore:

$$
x=F^{-1}(G(u)) .
$$

If $u$ is distributed uniformly in [0,1] one has:

$$
G(u)=u \quad \text { and } \quad x=F^{-1}(u) .
$$

This is the general rule which is used to obtain a random variable, $x$, which follows a specified sum distribution $F(x)$.

\section{Application to the Selection of Sampling Lines}

The definition of a sampling line requires the selection of a point $P$ in the associated sphere of a transfer point. The sampling line is then the line parallel to the $z$-axis through $P$. 
The selection of a point in a sphere of radius $r$, whose center is taken as the origin amounts to the random selection of three independent polar coordinates $\varrho, \theta$, $\phi$ with the following densities:

$$
p_{1}(\theta)=1 / 2 \pi ; \quad p_{2}(\phi)=(\sin \phi) / 2 ; \quad p_{3}(\varrho)=3 \varrho^{2} / r^{3} .
$$

In the present application it is however sufficient to select only the two coordinates of the projection of $P$ into a plane orthogonal to the $z$-axis. If the coordinates, $(s, \theta)$ are again in a polar system one has the following densities

$$
\begin{aligned}
& p_{1}(\theta)=1 / 2 \pi \\
& p_{2}(s)=3 s \sqrt{r^{2}-s^{2}} / r^{3}
\end{aligned}
$$

and the corresponding sum distributions:

$$
\begin{aligned}
& P_{1}(\theta)=\theta / 2 \pi \\
& P_{2}(s)=1-\left(1-s^{2} / r^{2}\right)^{3 / 2}
\end{aligned}
$$

accordingly $\theta$ and $s$ are obtained in the following way from a random variable, $u$, which is uniformly distributed:

$$
\begin{aligned}
& \theta=2 \pi u \\
& s=r \sqrt{1-u^{2 / 3}} .
\end{aligned}
$$

\section{References}

1. Chmelevsky, D.: Distributions et moyennes des grandeurs microdosimétriques à l'échelle du nanomètre. - Méthodes de Calcul et résultats. Rapport CEA-R-4785, Service de DocumentationCEN-Saclay, B.P. no. 2, 91190 Gif sur Yvette, France 1976

2. Fano, U.: Platzman's analysis of the delivery of radiation energy to molecules. Radiat. Res. 64, 217-232 (1975)

3. Glass, W. A., Toburen, L. H., Wilson, W. E., Roesch, W. D.: Energy ejected in fast proton collisions. In: Microdosimetry, Proc. 3rd Symposium on Microdosimetry, pp. 71-88 (Ebert, H. G., Ed.). Euratom, 4810 d-f-e, Brussels 1971

4. Hodges, D. C., Delafield, H. J., Dennis, J. A.: Preliminary measurements using a low pressure cloud chamber of the ranges of low energy electrons. In: Microdosimetry, Proc. 3rd Symposium on Microdosimetry, pp. 455-467 (Ebert, H. G., Ed.). Euratom, 4810 d-f-e, Brussels 1971

5. ICRU: Linear Energy Transfer, Report 16. Washington, D.C.: International Commission of Radiation Units and Measurements 1970

6. Inokuti, M.: Inelastic collisions of fast charged particles with atoms and molecules. - The Bethe theory revisited. Rev. Mod. Phys. 43, 297-347 (1971)

7. Kellerer, A. M., Chmelevsky, D.: Concepts of microdosimetry. I. Quantities. Rad. and Environm. Biophys. 12, 61-69 (1975)

8. Kellerer, A. M., Chmelevsky, D.: Concepts of microdosimetry. II. Probability distributions of the microdosimetric variables. Rad. and Environm. Biophys. 12, 1-12 (1975)

9. Kellerer, A. M., Chmelevsky, D.: Concepts of microdosimetry. III. Mean values of the microdosimetric distributions. Rad. and Environm. Biophys. 12, 321-335 (1975)

10. Kim, Y. K.: Energy distribution of secondary electrons. I. Consistency of experimental data. Radiat. Res. 61, 21-35 (1975)

11. Lea, D. E.: Actions of radiation of living cells. Cambridge: Cambridge University Press 1946 
12. Paretzke, H. G.: Comparison of track structure calculations with experimental results. In: Microdosimetry. Proc. 4th Symposium on Microdosimetry, pp. 141-164 (Ebert, H. G., Ed.). Euratom, 5122 d-e-f, Brussels 1974

13. Paretzke, H. G., Leuthold, G., Burger, G., Jacobi, W.: Approaches to physical track structure calculations. In: Microdosimetry. Proc. 4th Symposium on Microdosimetry, pp. 123-138 (Ebert, H. G., Ed.). Euratom, 5122 d-e-f, Brussels 1974

14. Patau, J. P., Malbert, M., Terrissol, M., Commanay, L.: Etudes dosimétriques dans des cavités sphériques situées en milieu semi-infini, irradié par des électrons de $2 \mathrm{MeV}$. In: Microdosimetry, Proc. 4th Symposium on Microdosimetry, pp. 755-775 (Ebert, H. G., Ed.). Euratom, 5122 d-e-f, Brussels 1974

15. Rossi, H. H.: Specification of radiation quality. Radiat. Res. 10, 522-531 (1959)

16. Rossi, H. H.: Distribution of radiation energy in the cell. Radiology 78, 530-535 (1962)

17. Rossi, H. H.: Energy distribution in the absorption of radiation. In: Advances in biological and medical physics, Vol. 11, pp. 27-85. London: Academic Press 1967

18. Rossi, H. H.: Biophysical implications of radiation quality. Radiat. Res. 59, 994-997 (1974)

19. Rossi, H. H., Biavati, M. H., Gross, W.: Local energy density in irradiated tissues. I. Radiobiological significance. Radiat. Res, 15, 431-439 (1961)

20. Terrissol, M., Patau, J. P.: Simulation du transport d'électrons d'énergie inférieure à un keV par une méthode de Monte-Carlo. In: Microdosimetry, Proc. 4th Symposium on Microdosimetry, pp. 717-730 (Ebert, H. G., Ed.). Euratom, 5122 d-e-f, Brussels 1973

21. Wilson, W. E.: Proton energy deposition distributions for small sites. Radiat. Res. 59, 117 abstract (1974)

Received March 21, 1977 